\title{
Arcwise connected attractors of infinite iterated function systems
}

\author{
Dan Dumitru
}

\begin{abstract}
The aim of this paper is to give a sufficient condition for the attractor of an infinite iterated function system to be arcwise connected.
\end{abstract}

\section{Introduction}

Iterated function systems (IFSs) were introduced in their present form by John Hutchinson ([7]) and popularized by Michael Barnsley ([1]). Also, infinite iterated function systems (IIFSs) were first mention in ([21]) and later studied in ([8], [9], [11], [13], [15], [16], [17]). The theory of noncompact attractors of infinite iterated function systems seems to be much less developed; only some attempts to unify finite and infinite systems based on the notion of multifunction were present, e.g., ([12], [14]). R. Miculescu and A. Mihail ([18], $[20])$ provided a general framework where attractors are nonempty closed and bounded subsets of complete metric spaces and where the iterated function systems may be infinite. In fact, in ([18]) it is proved that the Lipscomb's space is the attractor of an (IIFS). In ([19]) it is studied the shift space associated to an attractor of an infinite iterated function system. I. Chitescu and R. Miculescu ([2]) presented an example of a fractal, generated by Hutchinson's procedure, embedded in an infinite dimensional Banach space and its finite dimensional approximations.

Key Words: attractor, infinite iterated function system, arcwise connected, Lipscombs space.

2010 Mathematics Subject Classification: 28A80

Received: December 2012

Revised: February 2013

Accepted: March 2013 
In this paper we establish a sufficient condition for the attractor of an infinite iterated function system to be arcwise connected. We start with some notations: for a nonempty set $X$ we will denote by $\mathcal{P}(X)$ the set of nonempty subsets of $X$, by $\mathcal{K}(X)$ the set of nonempty compact subsets of $X$ and by $\mathcal{B}(X)$ the set of nonempty bounded closed subsets of $X$.

Definition 1.1. Let $(X, d)$ be a metric space. The Hausdorff-Pompeiu semidistance is the application $h: \mathcal{P}(X) \times \mathcal{P}(X) \longrightarrow[0,+\infty]$ defined by $h(A, B)=\max \{d(A, B), d(B, A)\}$, where

$$
d(A, B)=\sup _{x \in A} d(x, B)=\sup _{x \in A}\left(\inf _{y \in B} d(x, y)\right) .
$$

Theorem 1.1. ([1]) Let $(X, d)$ be a metric space and $h: \mathcal{P}(X) \times \mathcal{P}(X) \longrightarrow$ $[0, \infty]$ the Hausdorff-Pompeiu semidistance. Then:

1). $(\mathcal{B}(X), h)$ and $(\mathcal{K}(X), h)$ are metric spaces with $(\mathcal{K}(X), h)$ closed in $(\mathcal{B}(X), h)$.

2). If $(X, d)$ is complete then $(\mathcal{B}(X), h)$ and $(\mathcal{K}(X), h)$ are complete metric spaces.

In this paper, by $\mathcal{K}(X)$ and $\mathcal{B}(X)$ we will refer to $(\mathcal{K}(X), h)$ and $(\mathcal{B}(X), h)$.

Definition 1.2. Let $(X, d)$ be a metric space. For a function $f: X \longrightarrow X$ let us denote by $\operatorname{Lip}(f) \in[0,+\infty]$ the Lipschitz constant associated to $f$, which is $\operatorname{Lip}(f)=\sup _{x, y \in X ; x \neq y} \frac{d(f(x), f(y))}{d(x, y)}$. We say that $f$ is a Lipschitz function if $\operatorname{Lip}(f)<+\infty$ and a contraction if $\operatorname{Lip}(f)<1$.

Proposition 1.1. ([1]) Let $(X, d)$ be a metric space.

1). If $H$ and $K$ are two nonempty subsets of $X$ then $h(H, K)=h(\bar{H}, \bar{K})$, then

2). If $\left(H_{i}\right)_{i \in I}$ and $\left(K_{i}\right)_{i \in I}$ are two families of nonempty subsets of $X$

$$
h\left(\cup_{i \in I} H_{i}, \cup_{i \in I} K_{i}\right)=h\left(\bar{\bigcup}_{i \in I} H_{i}, \overline{\bigcup_{i \in I} K_{i}}\right) \leq \sup _{i \in I} h\left(H_{i}, K_{i}\right) .
$$

Definition 1.3. A family of continuous functions $\left(f_{i}\right)_{i \in I}, f_{i}: X \longrightarrow X$ for every $i \in I$, is said to be bounded if for every bounded set $A \subset X$ the set $\cup_{i \in I} f_{i}(A)$ is bounded.

Definition 1.4. a). An iterated function systems (IFS) consists on a finite family of contractions on a complete metric space. 
b). An infinite iterated function system (IIFS) on $X$ consists of a bounded family of continuous functions $\left(f_{i}\right)_{i \in I}$ on $X$ and it is denoted by $\mathcal{S}=\left(X,\left(f_{i}\right)_{i \in I}\right)$.

Definition 1.5. For an infinite iterated function system, $\mathcal{S}=\left(X,\left(f_{i}\right)_{i \in I}\right)$, the fractal operator, $F_{\mathcal{S}}: \mathcal{B}(X) \longrightarrow \mathcal{B}(X)$ is the function defined by $F_{\mathcal{S}}(B)=$ $\overline{\bigcup_{i \in I} f_{i}(B)}$ for every $B \in \mathcal{B}(X)$.

Remark 1.1. Let $\mathcal{S}=\left(X,\left(f_{i}\right)_{i \in I}\right)$ be an infinite iterated function system. If the functions $f_{i}$ are contractions for every $i \in I$, with $\sup \operatorname{Lip}\left(f_{i}\right)<1$ then the function $F_{\mathcal{S}}$ is a contraction that verifies $\operatorname{Lip}\left(F_{\mathcal{S}}\right) \leq \sup _{i \in I} \operatorname{Lip}\left(f_{i}\right)<1$.

Using Banach's contraction theorem we can prove the following:

Theorem 1.2. ([16]) Let $(X, d)$ be a complete metric space and $\mathfrak{S}=$ $\left(X,\left(f_{i}\right)_{i \in I}\right)$ an infinite iterated function system such that $c=\sup _{i \in I} \operatorname{Lip}\left(f_{i}\right)<1$. Then there exists a unique set $A \in \mathcal{B}(X)$ such that $F_{\mathcal{S}}(A)=A$. The unique set $A \in \mathcal{B}(X)$ is called the attractor of the infinte iterated function system $\mathcal{S}$. Moreover, for any $H_{0} \in \mathcal{B}(X)$ the sequence $\left(H_{n}\right)_{n \geq 0}$ defined by $H_{n+1}=F_{\mathcal{S}}\left(H_{n}\right)$ is convergent to $A$. For the speed of the convergence we have the following estimate: $h\left(H_{n}, A\right) \leq \frac{c^{n}}{1-c} h\left(H_{0}, H_{1}\right)$ for every $n \geq 1$.

Definition 1.6. Let $(X, d)$ be a metric space and $\left(A_{i}\right)_{i \in I}$ a family of nonempty subsets of $X$. The family $\left(A_{i}\right)_{i \in I}$ is said to be connected if for every $i, j \in I$ there exists $\left(i_{k}\right)_{k=\overline{1, n}} \subset I$ such that $i_{1}=i, i_{n}=j$ and $A_{i_{k}} \cap A_{i_{k+1}} \neq \emptyset$ for every $k \in\{1, \ldots, n-1\}$.

Definition 1.7. A metric space $(X, d)$ is called arcwise connected if for every $x, y \in X$ there exists a continuous function $\varphi:[0,1] \longrightarrow X$ such that $\varphi(0)=x$ and $\varphi(1)=y$.

\section{Main results}

Concerning the connectedness of the attractors of iterated function systems, we have the following result which is not true for an infinte iterated function system as one can in $([3])$ :

Theorem 2.1. ([5], [6], [7]) Let $(X, d)$ be a complete metric space and $\mathcal{S}=\left(X,\left(f_{i}\right)_{i=\overline{1, n}}\right)$ an iterated function system with $c=\max _{i=\overline{1, n}} \operatorname{Lip}\left(f_{i}\right)<1$. We 
denote by $A$ the attractor of $\mathcal{S}$ and by $A_{i}=f_{i}(A)$ for every $i \in\{1, \ldots, n\}$. Then the followings are equivalent:

1). The family of sets $\left(A_{i}\right)_{i=\overline{1, n}}$ is connected.

2). $A$ is arcwise connected.

3). $A$ is connected.

We give now a sufficient condition for the attractor of an infinite iterated function system to be arcwise connected.

Theorem 2.2. Let $(X, d)$ be a complete metric space and $\mathcal{S}=\left(X,\left(f_{i}\right)_{i \in I}\right)$ an infinite iterated function system with $c=\sup _{i \in I} \operatorname{Lip}\left(f_{i}\right)<1$. We denote by $A$ the attractor of $\mathcal{S}$ and by $A_{i}=f_{i}(A)$ for every $i \in I$. If the family of sets $\left(A_{i}\right)_{i \in I}$ is connected and $A=\cup_{i \in I} A_{i}$ then $A$ is arcwise connected.

Proof: We consider the following set of functions:

$$
\begin{gathered}
N=\{f: A \times A \times[0,1] \longrightarrow A \mid f(x, y, 0)=x, f(x, y, 1)=y \text { for every } \\
(x, y) \in A \times A\}
\end{gathered}
$$

On $N$ we define the following metric: For every $f, g \in N, d_{N}(f, g)=$ sup $d(f(x, y, t), g(x, y, t))$. Since the set $A$ is complete, it follows $(x, y, t) \in A \times A \times[0,1]$

that the metric space $\left(N, d_{N}\right)$ is complete. Let $x, y \in A$ be two fixed points. Since $A=\cup_{i \in I} A_{i}$ and the family of sets $\left(A_{i}\right)_{i \in I}$ is connected, it follows that there exist $n(x, y) \in \mathbb{N}^{*}, i_{x, y}^{0}, i_{x, y}^{n(x, y)} \in I,\left\{i_{x, y}^{k}\right\}_{k=\overline{0, n(x, y)}} \subset I$ and $\left\{p_{x, y}^{k}\right\}_{k=\overline{0, n(x, y)}} \subset A$ such that $x \in A_{i_{x, y}^{0}}, y \in A_{i_{x, y}^{n(x, y)}}, p_{x, y}^{0}=x, p_{x, y}^{n(x, y)}=y$ and $p_{x, y}^{k}, p_{x, y}^{k+1} \in A_{i_{x, y}^{k}}$ for every $k \in\{0, \ldots, n(x, y)-1\}$. We fix $q_{x, y}^{k} \in f_{i_{x, y}^{-1}}^{-1}\left(p_{x, y}^{k}\right)$ and $r_{x, y}^{k} \in f_{i_{x, y}^{k}}^{-1}\left(p_{x, y}^{k+1}\right)$ for every $k \in\{0, \ldots, n(x, y)-1\}$. For every $f \in N$ we define the following function which also belongs to $N: T f(x, y, t)=$ $f_{i_{x, y}^{k}}\left(f\left(q_{x, y}^{k}, r_{x, y}^{k}, n(x, y) t-k\right)\right)$ for every $t \in\left[\frac{k}{n(x, y)}, \frac{k+1}{n(x, y)}\right]$ and $k \in\{0, \ldots, n(x, y)-1\}$.

$T f$ is well-defined since on one hand $f_{i_{x, y}^{k}}\left(f\left(q_{x, y}^{k}, r_{x, y}^{k}, 1\right)\right)=f_{i_{x, y}^{k}}\left(r_{x, y}^{k}\right)=$ $p_{x, y}^{k+1}$ and on the other hand $f_{i_{x, y}^{k+1}}\left(f\left(q_{x, y}^{k+1}, r_{x, y}^{k+1}, 0\right)\right)=f_{i_{x, y}^{k+1}}\left(q_{x, y}^{k+1}\right)=p_{x, y}^{k+1}$. We will denote by $T^{m}=T \circ T \circ \ldots \circ T$ the $m$-times composition of $T$. We will denote by $\omega_{t}(f)=\lim _{\varepsilon \longrightarrow 0} \sup _{x, y \in(t-\varepsilon, t+\varepsilon)} d(f(x), f(y))$ the oscilation of $f$ in $t \in[0,1]$, by $f_{x, y}(t)=f(x, y, t)$ and by $\Omega(T f)=\sup _{(x, y, t) \in A \times A \times[0,1]} \omega_{t}\left(T f_{x, y}\right)$. Thus, for every $f, h \in N$ and $(x, y, t) \in A \times A \times[0,1]$ we have that: 


$$
\begin{gathered}
d(T f(x, y, t), T h(x, y, t))= \\
d\left(f_{i_{x, y}^{k}}\left(f\left(q_{x, y}^{k}, r_{x, y}^{k}, n(x, y) t-k\right)\right), f_{i_{x, y}^{k}}\left(h\left(q_{x, y}^{k}, r_{x, y}^{k}, n(x, y) t-k\right)\right)\right) \leq \\
\left.\left.\operatorname{Lip}\left(f_{i_{x, y}^{k}}^{k}\right) \cdot d\left(f\left(q_{x, y}^{k}, r_{x, y}^{k}, n(x, y) t-k\right)\right), h\left(q_{x, y}^{k}, r_{x, y}^{k}, n(x, y) t-k\right)\right)\right) \leq \\
\left.\left.c \cdot d\left(f\left(q_{x, y}^{k}, r_{x, y}^{k}, n(x, y) t-k\right)\right), h\left(q_{x, y}^{k}, r_{x, y}^{k}, n(x, y) t-k\right)\right)\right) \leq \\
c \cdot \sup _{(x, y, t) \in A \times A \times[0,1]} d(f(x, y, t), g(x, y, t))=c \cdot d_{N}(f, h) .
\end{gathered}
$$

Taking now the supremum after $(x, y, t) \in A \times A \times[0,1]$ we obtain that $d_{N}(T f, T h) \leq c \cdot d_{N}(f, h)$. From the Banach fixed point theorem there exists $\varphi \in N$ such that $T \varphi=\varphi$ and $T^{m} f \stackrel{m \rightarrow \infty}{\longrightarrow} \varphi$ for every $f \in N$. We will prove that $\varphi_{x, y}$ is continuous on $[0,1]$. We have $\Omega(T f) \leq \sup _{(x, y, t) \in A \times A \times[0,1]} \operatorname{Lip}\left(f_{i_{x, y}^{k}}\right)$. $\omega_{t}\left(f_{x, y}\right) \leq c \cdot \Omega(f)$

Thus, inductively we obtain that $\Omega\left(T^{m} f\right) \leq c^{m} \cdot \Omega(f) \stackrel{m \rightarrow \infty}{\longrightarrow} 0$. Hence $\Omega(\varphi)=0$ and so $\varphi$ is continuous with respect to $t$. Since $\varphi_{x, y}$ is a continuous function joining $x$ and $y$, it folows that $A$ is arcwise connected.

Example 2.1. We consider the following set in the plane $\mathbb{R}^{2}$ endowed with the euclidean metric $A=Q \cup\left(\cup_{n \geq 1} M_{n}\right)$, where $Q$ is the boundary of the square determinated by the vertices $\{(0,0),(1,0),(1,1),(0,1)\}$ and $M_{n}=$ $\left\{\frac{1}{2^{n}}\right\} \times[0,1]$ for every $n \in \mathbb{N}$. Then $A$ is the attractor of the countable iterated function systems $\mathcal{S}=\left(A,\left\{f_{n}, g_{n}\right\}_{n \in \mathbb{N}}\right)$ where the functions $f_{n}$ and $g_{n}$ are defined for every $n \in \mathbb{N}$ as follows: $f_{0}(x, y)=\left(0, \frac{y}{2}\right), g_{0}(x, y)=\left(0, \frac{y+1}{2}\right)$, $f_{1}(x, y)=\left(1, \frac{y}{2}\right), g_{1}(x, y)=\left(1, \frac{y+1}{2}\right), f_{2}(x, y)=\left(\frac{x}{2}, 0\right), g_{2}(x, y)=\left(\frac{x+1}{2}, 0\right)$, $f_{3}(x, y)=\left(\frac{x}{2}, 1\right), g_{3}(x, y)=\left(\frac{x+1}{2}, 1\right)$ and $f_{n}(x, y)=\left(\frac{1}{2^{n-3}}, \frac{y}{2}\right), g_{n}(x, y)=$ $\left(\frac{1}{2^{n-3}}, \frac{y+1}{2}\right)$ for every $n \geq 4$.

Moreover, we have that $A=\cup_{n \geq 0}\left(f_{n}(A) \cup g_{n}(A)\right)$ and the family of sets $\left(A_{n}\right)_{n \in \mathbb{N}}$ is connected, where $A_{2 n}=f_{n}(A)$ and $A_{2 n+1}=g_{n}(A)$ for every $n \in \mathbb{N}$. Thus $A$ is an arcwise connected set.

Example 2.2. We consider the following set in the plane $\mathbb{R}^{2}$ endowed with the euclidean metric:

$$
\begin{gathered}
A=(\{0\} \times[0,1]) \cup([0,1] \times\{1\}) \cup(\{1\} \times[0,1]) \cup \\
\left(\cup_{k \geq 0}\left[\frac{1}{2^{2 k+1}}, \frac{1}{2^{2 k}}\right] \times\{0\}\right) \cup\left(\cup_{k \geq 1}\left\{\frac{1}{2^{k}}\right\} \times\left[0, \frac{1}{2}\right]\right) \cup\left(\cup_{k \geq 1}\left[\frac{1}{2^{2 k+1}}, \frac{1}{2^{2 k}}\right] \times\left\{\frac{1}{2}\right\}\right) .
\end{gathered}
$$

Then $A$ is the attractor of the countable iterated function system $\mathcal{S}=$ $\left(A,\left\{f_{n}\right\}_{n \geq 0}\right)$, where the functions $f_{n}: A \longrightarrow A, n \in \mathbb{N}$ are defined by: $f_{0}(x, y)=\left(0, \frac{y}{2}\right), f_{1}(x, y)=\left(0, \frac{y+1}{2}\right), f_{2}(x, y)=\left(\frac{x}{2}, 1\right), f_{3}(x, y)=\left(\frac{x+1}{2}, 1\right)$, 
$f_{4}(x, y)=\left(1, \frac{y+1}{2}\right), f_{5}(x, y)=\left(1, \frac{y}{2}\right)$ and for $n \geq 6$ we consider that $f_{n}(x, y)=$ $\left(\frac{x+1}{2^{2 k-2}}, \frac{1}{2}\right)$ if $n=4 k$ and $k \geq 2, f_{n}(x, y)=\left(\frac{1}{2^{2 k-2}}, \frac{y}{2}\right)$, if $n=4 k+1$ and $k \geq 2$, $f_{n}(x, y)=\left(\frac{x+1}{2^{2 k-1}}, 0\right)$, if $n=4 k+2$ and $k \geq 1, f_{n}(x, y)=\left(\frac{1}{2^{2 k-1}}, \frac{y}{2}\right)$, if $n=4 k+3$ and $k \geq 1$.

Moreover, it follows that $A=\cup_{n \geq 0} f_{n}(A)$ and the family of sets $\left(A_{n}\right)_{n \in \mathbb{N}}$ is connected. Thus $A$ is arcwise connected.

Example 2.3. (Lipscomb's space) [For more details see ([18], [19], $[20])]$.

Let $A$ be an arbitrary set and for $z \in A$ we denote by $A^{\prime}=A-\{z\}$. The points of $l^{2}(A)$ are the collections of real numbers indexed by the points of $A$. The topology of $l^{2}(A)$ is induced from the metric $d(x, y)=\sqrt{\sum_{a}\left(x_{a}-y_{a}\right)^{2}}$, where we think $x_{a}$ as the $a$-th coordinate of $x$. Let us also consider, for the case when $A$ is an arbitrary set with the discrete topology, the Baire space $N(A)$ which is the topological product of countably many copies $A_{n}$ of $A$. Hence the points of $N(A)$ consist of all the sequences $a=a_{1} a_{2} \ldots a_{n} \ldots$, with $a_{n} \in A$. The Lipscomb's space $L(A)$ is a quotient space of $N(A)$ such that each equivalence class consists of either a single point or two points. Those classes with two points come from identifying $a_{1} a_{2} \ldots a_{k-2} a_{k-1} a_{k} a_{k} \ldots$ with the point $a_{1} a_{2} \ldots a_{k-2} a_{k} a_{k-1} a_{k-1} \ldots$, where $a_{k-1} \neq a_{k}$. Let $\varpi^{A}$ be the imbedded version of $L(A)$ endowed with the induced topology of $l^{2}(A)$. More precisely, let us consider the function $s: L(A) \longrightarrow l^{2}(A)$ given by $s(\alpha)=\left(\alpha_{b}\right)_{b \in A}$, where $\alpha=a_{1} a_{2} \ldots \in N(A)$ and

$$
\alpha_{b}=\left\{\begin{array}{cc}
\sum_{k \text { with } a_{k}=b} \frac{1}{2^{k}}, & \text { if there exists } k \text { such that } a_{k}=b \\
0, & \text { if there exists no } k \text { such that } a_{k}=b .
\end{array}\right.
$$

Then $\varpi^{A}$ is $s(L(A))$. Let us consider now the complete metric space $\left(l^{2}(A), d\right)$. For each $a \in A$, let $f_{a}: l^{2}(A) \longrightarrow l^{2}(A)$ be the function given by $f_{a}(x)=\frac{1}{2}\left(x+u_{a}\right)$ for all $x \in l^{2}(A)$, where $u_{z}=0_{l^{2}(A)}$ and for every $a \in A \backslash\{z\}, u_{a}=\left(\alpha_{j}\right)_{j \in A} \in l^{2}(A)$ is described by $\alpha_{j}=0$ for $j \neq a$ and $\alpha_{a}=1$. It is proved in $([18])$ that $\mathcal{S}=\left(l^{2}(A),\left(f_{a}\right)_{a \in A}\right)$ is an infinite iterated function system and the attractor of $\mathcal{S}$, denoted $\varpi^{A}$, is the imbedded version of Lipscomb's space $L(A)$ in $l^{2}(A)$. In ([20]), using some results from $([19])$, it was proved that $\varpi^{A}=\cup_{a \in A} f_{a}\left(\varpi^{A}\right)$. We consider now the fixed points $u_{a}$ and $u_{b}$ of $f_{a}$ and $f_{b}$. Then $f_{a}\left(u_{b}\right)=f_{b}\left(u_{a}\right)=\frac{1}{2}\left(u_{a}+u_{b}\right) \in f_{a}\left(\varpi^{A}\right) \cap f_{b}\left(\varpi^{A}\right)$ for every $a \neq b \in A$. Thus the family of sets $\left(A_{a}=f_{a}\left(\varpi^{A}\right)\right)_{a \in A}$ is connected. Hence, it follows from theorem 2.2, that $\varpi^{A}$ is arcwise connected.

Open Problem. In what conditions theorem 2.1. is valid for an infinite iterated function system? 
Aknowledgement. The author would like to thank the referee for his useful suggestions to improve the paper.

\section{References}

[1] M.F. Barnsley, Fractals everywhere, Academic Press Professional, Boston, 1993.

[2] I. Chiţescu, R. Miculescu, Approximation of fractals generated by Fredholm integral equations, J. Comput. Anal. Appl., Volume 11(2009), No. 2, 286-293.

[3] D. Dumitru, Topological properties of the attractors of iterated function systems, An. Şt. Univ. Ovidius Constanţa, Vol. 19(3), 2011, 117-126.

[4] R. Engelking, General topology, Polish Scientific Publishers.

[5] K.J. Falconer, The geometry of fractal sets, Cambridge University Press, Cambridge, 1986.

[6] M. Hata, On the structure of self-similar sets, Japan J. Appl. Math., 2(1985), 381-414.

[7] J. Hutchinson, Fractals and self-similarity, Indiana Univ. Math. J., 30(1981), No. 5, 713-747.

[8] J. Jachymski, G. Gwóźdź-Łukawska, The Hutchinson theory for infinite iterated function systems, Bull. Austral. Math. Soc., 72(2005), 441-454.

[9] B. Kieninger, Iterated function systems on compact Hausdorff spaces, Ph.D. Thesis, Berichte aus der Mathematik, Shaker-Verlag, Aachen 2002.

[10] J. Kigami, Analysis on fractals, Cambridge univ. Press, 2001.

[11] M. Kwiecinski, A locally connected continuum which is not an IFS attractor, IMUJ Preprint, 1998/14.

[12] A. Lasota, J. Myjak, Attractors of multifunctions, Bull. Pol. Ac. Sci.: Math., 48(2000), 319-334.

[13] K. Leśniak, Infinite iterated function systems: a multivalued approach, Bull. Pol. Acad. Sci. Math., 52(2004), No. 1, 1-8.

[14] K. Leśniak, Fixed points of the Barnsley-Hutchinson operators induced by hypercondensing maps, Le Matematiche, Vol. LX(2005), fasc. I, 67-80. 
[15] L. Máté, The Hutchinson-Barnsley theory for certain noncontraction mappings, Period. Math. Hungar., 27, No. 1, 1993, 21-33.

[16] R. D. Mauldin, M. Urbański, Dimensions and measures in infinite iterated function systems, Proc. London Math. Soc., (3) 73(1996), 105-154.

[17] R. D. Mauldin, M. Urbański, On the uniqueness of the density for the invatiant measure in an infinite hyperbolic iterated function system, Periodica Math. Hungarica, 37(1998), 47-53.

[18] R. Miculescu, A. Mihail, Lipscomb's space $\omega^{A}$ is the attractor of an infinite IFS containing affine transformations of $l^{2}(A)$, Proc. Amer. Math. Soc., 136(2008), 587-592.

[19] R. Miculescu, A. Mihail, The shift space for an infinite iterated function system, Math. Rep., 11(61)(2009), No. 1, 21-32.

[20] R. Miculescu, A. Mihail, Lipscomb's $L(A)$ space fractalized in $l^{p}(A)$, Mediterranean Journal of Mathematics, doi:10.1007/s00009-011-0133-9, 2011, 1-10.

[21] K. R. Wicks, Fractals and Hyperspaces, LNM 1492, Springer-Verlag, Berlin, 1991.

Dan DUMITRU

Spiru Haret University of Bucharest

Department of Mathematics and Computer Science

13 Ghica str., Bucharest, Romania

e-mail: dandumitru1984@yahoo.com 